\title{
Is There a Missing Galaxy Problem at High Redshift?
}

\section{Citation}

Nagamine, Kentaro, Renyue Cen, Lars Hernquist, Jeremiah P. Ostriker, and Volker Springel. 2004. "Is There a Missing Galaxy Problem at High Redshift?" The Astrophysical Journal 610 (1): 45-50. https://doi.org/10.1086/421379.

\section{Permanent link}

http://nrs.harvard.edu/urn-3:HUL.InstRepos:41381677

\section{Terms of Use}

This article was downloaded from Harvard University's DASH repository, and is made available under the terms and conditions applicable to Other Posted Material, as set forth at http:// nrs.harvard.edu/urn-3:HUL.InstRepos:dash.current.terms-of-use\#LAA

\section{Share Your Story}

The Harvard community has made this article openly available.

Please share how this access benefits you. Submit a story.

Accessibility 
DRAFT VERSION

\title{
Is There a Missing Galaxy Problem at High Redshift?
}

\author{
Kentaro Nagamine ${ }^{1}$, Renyue Cen $^{2}$, Lars Hernquist ${ }^{1}$, Jeremiah P. Ostriker ${ }^{2,3}$, \\ \& Volker Springel ${ }^{4}$
}

\begin{abstract}
We study the evolution of the global stellar mass density in a $\Lambda$ cold dark matter $(\Lambda \mathrm{CDM})$ universe using two different types of hydrodynamical simulations (Eulerian TVD and SPH) and the analytical model of Hernquist \& Springel (2003). We find that the theoretical calculations all predict both a higher stellar mass density at $z \sim 3$ than indicated by current observations, and that the peak of the cosmic star formation rate history should lie at $z \gtrsim 5$. Such a star formation history implies that as much as $(70 \%, 30 \%)$ of the total stellar mass density today must already have formed by $z=(1,3)$. Our results suggest that current observations at $z \sim 3$ are missing as much as $50 \%$ of the total stellar mass density in the Universe, perhaps owing to an inadequate allowance for dust obscuration in star-forming galaxies, limited sample sizes, or cosmic variance. We also compare our results with some of the updated semi-analytic models of galaxy formation.
\end{abstract}

Subject headings: cosmology: theory — stars: formation — galaxies: formation - galaxies: evolution - methods: numerical

\section{Introduction}

Is the evidence for high redshift galaxy formation consistent with the concordance $\Lambda \mathrm{CDM}$ model? Recent observational results include the discovery of Extremely Red Objects at $z \geq 1$ (e.g. Elston et al. 1988; McCarthy et al. 1992; Hu \& Ridgway 1994; Cimatti

\footnotetext{
${ }^{1}$ Harvard-Smithsonian Center for Astrophysics, 60 Garden Street, Cambridge, MA 02138, U.S.A. Email: knagamin@cfa.harvard.edu

${ }^{2}$ Princeton University Observatory, Princeton, NJ 08544, U.S.A.

${ }^{3}$ Institute of Astronomy, University of Cambridge, Madingley Road, Cambridge, CB3, OHA, UK

${ }^{4}$ Max-Planck-Institut für Astrophysik, Karl-Schwarzschild-Straße 1, 85740 Garching bei München, Germany
} 
et al. 2003; Smail et al. 2002), Sub-millimeter galaxies at $z \geq 2$ (e.g. Smail et al. 1997; Chapman et al. 2003), Lyman-break galaxies (LBGs) at $z \sim 3$ (e.g. Steidel et al. 1999), and galaxies at $z \gtrsim 4$ either by their Lyman- $\alpha$ emission (e.g. Hu et al. 1999; Rhoads \& Malhotra 2001; Taniguchi et al. 2003; Kodaira et al. 2003; Ouchi et al. 2003a) or by their optical to near infrared (IR) colors (e.g. Iwata et al. 2003; Ouchi et al. 2003b; Dickinson et al. 2003b).

Multiband photometry including the near-IR band makes it possible to estimate the stellar mass of these high redshift galaxies by fitting the observed photometric results with artificial galaxy spectra generated by a population synthesis model. Using this technique, several groups have now estimated the stellar mass density in the Universe in the redshift range of $0 \leq z \leq 3$ (e.g. Brinchmann \& Ellis 2000; Cole et al. 2001; Cohen 2002; Dickinson et al. 2003a; Fontana et al. 2003; Glazebrook et al. 2004). Rudnick et al. (2003) also estimated the stellar mass density at $z=0-3$ by combining the estimates of the rest-frame optical luminosity density and the mean cosmic mass-to-light ratio. These observational estimates constrain the evolution of the stellar mass density $\Omega_{\star}$ as a function of redshift or cosmic time.

The observations indicate the presence of a significant stellar population at high redshift, and, by comparing with semi-analytic models of galaxy formation, some authors claim that $\Lambda$ CDM models seriously underpredict galaxy formation at $z \sim 3$. For example, Fontana et al. (2003) compare their estimate of $\Omega_{\star}$ from the Hubble Deep Field (HDF) South with the semianalytic model of Menci et al. (2002), and argue that the high-mass tail of the galaxy stellar mass function is not adequately described by CDM models. Comparing to the same semianalytic model, Poli et al. (2003) argue that hierarchical models lack sufficient star formation at $z=2-4$, resulting in a failure to reproduce the pronounced brightening of the luminosity function at these redshifts. Also, Dickinson et al. (2003a) find that their data from the HDF North suggest a steeper increase in $\Omega_{\star}$ than some semi-analytic models (Kauffmann et al. 1999; Somerville et al. 2001; Cole et al. 2000), and some of the semi-analytic models predict higher stellar mass density compared to their data points. Given these contradicting claims and the large parameter space available to the semi-analytic models as well as the limitations of the current observational samples, it is not clear at present if any of these discrepancies actually pose a serious problem to hierarchical evolution.

In this paper, we compare the observational data with the results from state-of-theart cosmological simulations of the standard concordance $\Lambda$ CDM model and the theoretical model of Hernquist \& Springel (2003) (hereafter H\&S model), to show that, contrary to some of the claims, theory predicts a higher $\Omega_{\star}$ at $z=3$ than indicated by current observations and that the cosmic star formation rate (SFR) density peaks at $z \geq 5$, earlier than suggested by most semi-analytic models. Also, we will explicitly compare our results with those 
from the updated semi-analytic models by Somerville et al. (2001), Granato et al. (2000) (GALFORM), and Menci et al. (2002).

\section{Simulations}

We will show results from two different types of cosmological hydrodynamic simulations. Both approaches include "standard" physics such as radiative cooling/heating, star formation, and supernova (SN) feedback, although the details of the models and the parameter choices differ somewhat.

One set of simulations was performed using an Eulerian approach, which relies on a particle-mesh method for the gravity and the Total Variation Diminishing (TVD) method (Ryu et al. 1993) with a fixed mesh for the hydrodynamics. The treatment of the radiative cooling and heating is described in Cen (1992) in detail. The structure of the code is similar to that of Cen \& Ostriker $(1992,1993)$, but the code has significantly improved over the years with additional input physics. It has been used for a variety of studies, including the evolution of the intergalactic medium (Cen et al. 1994; Cen \& Ostriker 1999a,b), damped Lyman- $\alpha$ absorbers (Cen et al. 2003), and galaxy formation (e.g. Cen \& Ostriker 2000; Nagamine, Fukugita, Cen, \& Ostriker 2001a,b; Nagamine 2002).

Our other simulations were done using the Lagrangian Smoothed Particle Hydrodynamics (SPH) technique. We use an updated version of GADGET (Springel et al. 2001), which uses an 'entropy conserving' formulation (Springel \& Hernquist 2002) to mitigate problems with energy/entropy conservation (e.g. Hernquist 1993) and overcooling. This code also uses a multiphase model of the interstellar medium to describe self-regulated star formation and a phenomenological model for galactic winds (Springel \& Hernquist 2003a). This approach has been used to study the evolution of the cosmic SFR (Springel \& Hernquist 2003b), damped Lyman- $\alpha$ absorbers (Nagamine, Springel, \& Hernquist 2004a,b), and galaxies at high redshifts (Nagamine, Springel, Hernquist, \& Machacek 2004).

The cosmological parameters adopted in the simulations are intended to be consistent with recent observational determinations (e.g. Spergel et al. 2003), as summarized in Table 1.

\section{Stellar Mass Density}

Figure 1 shows the evolution of the global stellar mass density $\Omega_{\star}$ as a function of redshift. Figure 1a compares the simulation results with the observations, and Figure $1 \mathrm{~b}$ compares the semi-analytic models with the observations. Our results are scaled to unity at 
$z=0$ (see discussion below). Observational data points are normalized to the local estimate by Cole et al. (2001, filled circle at $z=0$ ) following Rudnick et al. (2003). The result of the H\&S model shown in both panels is simply the integral of their approximate formula for the SFR which will be given in Equation (1).

For recent epochs $(z \lesssim 1)$, the computed results are within the range of the observational data. However, the important result here is that at $1 \lesssim z \lesssim 3$, all the observational estimates (both corrected and uncorrected estimates for the incompleteness of the survey) are smaller than the simulation results by more than a factor of two. The early development of the stellar mass density is much faster in the simulations than is suggested by current observations. As we discuss in Section 4 , the larger $\Omega_{\star}$ at $z \sim 3$ originates from a higher SFR at $z \gtrsim 3$ which peaks at $z \gtrsim 5$ in both the TVD and SPH simulations. Such a star formation history implies that about $70 \%(50,30,15 \%)$ of the total stellar mass density today must have been in place by $z=1(z=2,3,5)$. The contribution to $\Omega_{\star}(z=0)$ from the star formation at $z>6$ is about $10 \%$.

We note that the data points of Rudnick et al. (2003, magenta filled squares) should be considered as a lower limit, as they did not attempt to make any extrapolations to correct for the incompleteness of their data. On the other hand, Dickinson et al. (2003a, green square boxes) correct for the incompleteness of their data by integrating the Schechter fit with a faint-end slope of $\alpha=-1.4$ down to a fainter magnitude. For the Fontana et al. (2003, blue crosses) data points, we have plotted the values for the SMC extinction case (which yields slightly larger $\Omega_{\star}$ values compared to the Calzetti et al. (2000) extinction), and the upper error bars are extended up to their 'maximum mass' estimates. For the Glazebrook et al. (2004, black filled triangles) data points, we plotted the values for the mass limit of $\log \left(M / M_{\odot}\right)=10.2$ which yields largest $\Omega_{\star}$ estimates from their data. We also note that dust extinction is taken into account in the analyses by Brinchmann \& Ellis (2000); Dickinson et al. (2003a); Fontana et al. (2003); Glazebrook et al. (2004) by allowing the extinction parameter to vary when fitting the broadband photometric measurements of each galaxy by the spectral energy distribution generated by a population synthesis model. Rudnick et al. (2003) adopt the Calzetti extinction with $E(B-V)=0.30$ when deriving the mean mass-to-light ratio from the rest-frame $U-V$ color.

The scaling of the simulation results to the local estimate at $z=0$ leaves us with some concerns, because the relative value of $\Omega_{\star}$ does indeed depend on this scaling. However, some kind of normalization is necessary to compare the results of different numerical models on the same basis, because every numerical model has its input parameters, and what we would like to focus in this paper is the relative speed of the development of $\Omega_{\star}$ from high redshift to the present time. The two TVD simulations in fact yield different values of $\Omega_{\star}$ owing to cosmic 
variance and differences in the set of cosmological parameters adopted in the simulations: $\Omega_{\star}=0.0077$ and 0.0052 for N864L22 and N768L25, respectively. The normalization of $\Omega_{\star}$ at $z=0$ in Figure 1 can be partially explained by the scaling of $\Omega_{\star}$ with the baryon mass density in the different simulations. Hernquist \& Springel (2003) have shown that $\Omega_{\star}$ scales as $\Omega_{\star} \sim \Omega_{b}^{1.8}$ from theoretical arguments, and Gardner et al. (2003) found that the amount of cold gas and stars in their SPH simulations follow $\Omega_{\star} \propto \Omega_{b}^{1.0-2.0}$.

Using the cosmological parameters of the two TVD runs, the scaling $\Omega_{\star} \propto \Omega_{b}^{1.8}$ gives the expected ratio $\Omega_{\star}(\mathrm{N} 864 \mathrm{~L} 22) / \Omega_{\star}(\mathrm{N} 768 \mathrm{~L} 25)=1.7$. The actual corresponding ratio from the two simulations is $(0.0077 / 0.0052)=1.5$, which is a reasonable agreement, given other uncertainties, such as cosmic variance. It is expected that the H\&S model (blue long-dashed line in Figure 1) should yield the highest $\Omega_{\star}$ at $z=3$, because it is intended to remove the effects of limited resolution and cosmic variance. Since the results of N864L22 and N768L25 are not corrected for the limited box-size and resolution, it is natural that they lack the earliest star formation at $z>10$, resulting in a lower $\Omega_{\star}$ at $z=3$. This is also true of the SPH G6 run which by itself cannot resolve the entire starforming population at $z \gtrsim 3$. Also, a comparison of the N864L22 and N768L25 results gives an idea of the level of cosmic variance for a volume of $\approx\left(25 h^{-1} \mathrm{Mpc}\right)^{3}$, and the deviation of the SPH G6 run from the $\mathrm{H} \& \mathrm{~S}$ model is also a consequence of cosmic variance.

Figure 1b compares the results of $\mathrm{H} \& \mathrm{~S}$ theoretical model, semi-analytic models of Somerville et al. (2001), Granato et al. (2000), Menci et al. (2002), and observations. The model shown for Somerville et al. (2001) is the updated 'accelerated quiescent' model which was also used in Somerville et al. (2004), and follows the merger tree down to halos with circular velocity $30 \mathrm{~km} \mathrm{~s}^{-1}$. The result shown for Menci et al. (2002) is the original model used by Poli et al. (2003) and Fontana et al. (2003) for the comparison to their observational data. Recently Menci et al. (2003) updated their model to include a starburst mode of star formation, which gives a result close to that of GALFORM. The results of the semi-analytic models are somewhat higher than the observational estimates, but within the upper limit of Fontana et al. (2003) data point at $z=3$. It is also clear that the predicted $\Omega_{\star}$ by the H\&S model is higher than that of the two semi-analytic models by about a factor of two. The discrepancy between the models and observations increases at $z \sim 1.5$, and is significant at more than $1-\sigma$.

\section{Cosmic Star Formation Rate at $0 \lesssim z \lesssim 6$}

In Figure 2, we show the cosmic SFR density as a function of redshift. Panel (a) shows the simulation results, and panel (b) shows the semi-analytic model results. The 
simulation results shown in this figure are extracted directly from the runs, without any further adjustments. The H\&S model that is shown in both panels has an approximate form as

$$
\dot{\rho}_{\star}=\dot{\rho}_{\star}(0) \frac{\chi^{2}}{1+\alpha(\chi-1)^{3} \exp \left(\beta \chi^{7 / 4}\right)},
$$

where $\chi(z) \equiv\left(H(z) / H_{0}\right)^{2 / 3}$. For a $\Lambda$ CDM universe with the star formation and feedback algorithm described by Springel \& Hernquist (2003a), the parameters defining the SFR density take the values $\alpha=0.012, \beta=0.041$, and $\dot{\rho}_{\star}(0)=0.013 \mathrm{M}_{\odot} \mathrm{yr}^{-1} \mathrm{Mpc}^{-1}$. See Section 5 for further discussion on this formula.

The line types and data points are described in the caption. The observationally estimated ultra-violet (UV) luminosity densities $\rho_{\mathrm{UV}}$ have been converted into the SFR by $\rho_{\mathrm{UV}}\left[\mathrm{erg} \mathrm{s}^{-1} \mathrm{~Hz}^{-1} \mathrm{Mpc}^{-3}\right]=8.0 \times 10^{27} S F R\left[M_{\odot} \mathrm{yr}^{-1} \mathrm{Mpc}^{-3}\right]$ (Madau, Pozzetti, \& Dickinson 1998). The SFR data points are corrected for dust extinction according to a prescription similar to that of Steidel et al. (1999): we assume the highly uncertain extinction correction factors to be $1.3(z<2)$ and $4.0(z>2)$, while Steidel et al. (1999) used higher values 2.7 $(z<2)$ and $4.7(z>2)$.

It is important to note that each data point has been derived under different assumptions, because the faint-end slope of the luminosity function of galaxies at high redshift is not well constrained and adopting a steeper slope and integrating down to fainter magnitudes would certainly yield a larger UV luminosity density. Here we describe some of the high redshift data points. Steidel et al. (1999, open stars at $z=3,4$ ) derived their UV luminosity density by integrating the luminosity function with a faint-end slope of $\alpha=-1.6$ down to $0.1 L^{*}$. For their data points, we read off the SFR from Fig.9, corrected to our flat- $\Lambda$ cosmology, and applied our extinction correction. Giavalisco et al. (2004, open triangles at $z=3-6$ ) integrated the Schechter fit with a faint-end slope of $\alpha=-1.6$ down to $0.2 L_{3}^{*}$, where $L_{3}^{*}$ is the characteristic UV luminosity of LBGs at $z \sim 3$. Iwata et al. (2003, open pentagon at $z=5$ ) integrated their Schechter fit with $\alpha=-1.5$ in the magnitude range of $22.5<M_{\mathrm{UV}}-5 \log h<-20.0$. This integration range was chosen to match with the limiting magnitude of the $z \sim 4$ sample by Steidel et al. (1999) in terms of absolute magnitude, therefore it is expected from the shape of the luminosity function that the resultant UV luminosity density at $z \sim 5$ is $0.56-0.69$ times of that at $z \sim 3$ in the same absolute magnitude range, depending on the choice of cosmology and the integration range. For the Ouchi et al. (2003b, inverted open triangles) data points, we show their total UV luminosity density case where the luminosity function with a faint-end slope of $\alpha=-2.2$ is integrated down to $0.1 L^{*}$.

An important theoretical result here is that all the simulation results and the H\&S model peak at $z \geq 5$, and not at lower redshifts, as is often found in semi-analytic models of 
galaxy formation (e.g. Baugh et al. 1998; Kauffmann et al. 1999; Cole et al. 2000; Somerville et al. 2001, see panel (b)). Observationally, it is an unsettled problem whether the SFR levels off at high-redshift (e.g. Steidel et al. 1999) or still increases beyond $z>3$ (Lanzetta et al. 2002). In particular, Lanzetta et al. (2002) stress the importance of the cosmological surface

dimming effect and argue that previous measurements have missed a significant fraction of the ultraviolet luminosity density of the universe at $z \geq 2$. The scatter in the data points at $z>3$ is still large, and it is not possible at this time to determine the trend in SFR at these redshifts. However, our point is a theoretical one, and does not depend on the distribution of data points as we detail in Section 5 .

In passing, we note that the absolute values of $\Omega_{\star}$ at $z=0$ computed by integrating the SFR curves shown in Fig.2b are 0.0037, 0.0031, 0.0041, and 0.0090 for the models by Hernquist \& Springel (2003), Somerville et al. (2001), Granato et al. (2000), and Menci et al. (2002), respectively.

The result of TVD N864L22 at $z<1$ is slightly higher than most of the observational data points and has two large bumps at $z \sim 1.0$ and 2.0. These two peaks presumably owe to major merger events taking place in the simulation, and are a consequence of cosmic variance in the relatively small box utilized. If we had utilized a larger box, then we would expect the curve to be smooth. Clearly the box-size of $L_{\mathrm{box}}=20-30 h^{-1} \mathrm{Mpc}$ is not large enough to accurately model the volume averaged quantities in the universe at $z<1$.

\section{Discussion \& Conclusions}

We have shown that two independent different types of numerical hydrodynamic simulations both predict that the cosmic star formation rate density should peak at $z \geq 5$, and that this relatively early peak in the SFR leads to a more rapid development of the stellar mass density than current observational estimates. When all the results are scaled to the local value at $z=0$, the stellar mass density at $z=3$ in the simulations is larger than observed values by more than a factor of two. We also showed in Figure 1b that the semianalytic models of Somerville et al. (2001), Granato et al. (2000), and Menci et al. (2002) predict larger $\Omega_{\star}$ than current observational estimates, but within the upper error bar of the Fontana et al. (2003) data point at $z=3$. In particular, the H\&S model predicts higher $\Omega_{\star}$ than those semi-analytic models by about a factor of two at $z=3$. This comes from the fact that both the simulations and H\&S model has a peak of SFR at $z=5-7$, whereas the semi-analytic models have a peak at $z=2-4$.

The high $\Omega_{\star}$ predicted by our simulations and the model of Hernquist \& Springel (2003) 
suggest that current observations are missing nearly half of the total stellar mass density in the universe at high redshifts. This missing stellar mass could be hidden in a population of red galaxies that have not been detected previously in the optical ground-based data. In fact, Franx et al. (2003) and Daddi et al. (2004) find such a population of red galaxies at $z \geq 2$ in HDF-South that has a volume density half that of LBGs at $z=3$ and a stellar mass density comparable to that of LBGs. However, the data points by Rudnick et al. (2003) include this red galaxy population. Therefore the cosmic variance might be a stronger cause for the underestimate of $\Omega_{\star}$ by the current observations. This is hinted by the absence of this red population in HDF-North. If these red populations are strongly clustered as suggested by Daddi et al. (2003), observations with small fields-of-view could easily miss them. The stellar mass density in the simulations and observations agree reasonably well at $z<1$ where the rate of increase is much slower than at higher redshift. In addition, the dust extinction correction may seriously underestimate the fraction of early star formation that is heavily obscured. If we only had optical UV observations of nearby starburst galaxies - rather than the full spectrum extending to the submillimeter - we would greatly underestimate star formation in these systems (e.g. Hughes et al. 1998; Meurer et al. 1999; Barger et al. 2000; Calzetti et al. 2000; Takagi et al. 2003). Future studies of starforming galaxies in the far-infrared wavelengths (e.g. Kennicutt et al. 2003) using the Spitzer Space Telescope, in the submillimeter (e.g. Tecza et al. 2004), in the millimeter (e.g. Bekki \& Shioya 2000) using the Atacama Large Millimeter Array (ALMA), and in X-rays (e.g. Norman et al. 2004) using the Chandra X-ray Observatory would enable us to constrain the nature of starburst galaxies better, and estimate the intrinsic SFR more accurately.

It is reassuring that the two different sets of simulations, which use very different hydrodynamic methods (i.e. Eulerian TVD and SPH), both give a star formation history that peaks at $z \geq 5$. As argued by Hernquist \& Springel (2003) this is to be expected, because the evolution of the cosmic SFR is driven mainly by a competition between gravity and the expansion of the Universe, with a weaker dependence on the details of star formation and feedback. The form of equation (1) given in Section 4 can be understood as follows. At high redshifts, when the cooling time is short, star formation is limited primarily by the gravitational growth of halos, which is independent of the dissipative gas dynamics. Thus, the parameter $\beta$ and the factor $\chi^{7 / 4}$ in the exponential of equation (1) are determined by the form of the matter power spectrum, and the description of star formation and feedback enter into $\dot{\rho}_{\star}(z)$ only logarithmically. At low redshifts, the supply of star forming gas is limited by the expansion rate of the Universe, fixing the dependence of $\dot{\rho}_{\star}(z)$ on $\chi$ as $z \rightarrow 0$. The explicit influence of the prescription for star formation and feedback is again subdominant and mainly affects the values of the normalization parameters $\alpha$ and $\dot{\rho}_{\star}(0)$.

For these reasons, equation (1) can be generalized straightforwardly to other cosmologies 
and to include other physics (e.g. Yoshida et al. 2003). Moreover, as shown in e.g. figure 6 of Hernquist \& Springel (2003), the fact that the SFR density is regulated mainly by the competition between gravity and the expansion of the Universe means that the peak in $\dot{\rho}_{\star}(z)$ should lie at $z \approx 5$, unless an implausible value is adopted for the parameters governing star formation. Hence, it is not surprising that the various sets of simulations should be consistent, with residual differences owing to details in the cosmology and, most important, cosmic variance and resolution limitations. We plan to investigate these issues in the future using the algorithms described here, as well as adaptive mesh refinement codes.

For now, the agreement between our different numerical approaches supports the general arguments made by Hernquist \& Springel (2003), that the SFR density should peak at $z \geq 5$, mostly independent of the details of star formation and feedback. As we have demonstrated here, such an early peak in the cosmic star formation history yields $\Omega_{\star}$ that clearly exceeds current observational estimates and the results of semi-analytic models, suggesting that most of the stars in the universe at $z \gtrsim 3$ are "missing."

We thank Rachel Somerville, Carlton Baugh, Shaun Cole, and Nicola Menci for providing the result of their semi-analytic model. This work was supported in part by NSF grants ACI 96-19019, AST 98-02568, AST 99-00877, AST 00-71019, and AST-0206299, and NASA ATP grant NAG5-12140 and NAG5-13292, NAG5-13381. The SPH simulations were performed at the Center for Parallel Astrophysical Computing at Harvard-Smithsonian Center for Astrophysics. The TVD simulations were performed at the National Center for Supercomputing Applications (NCSA).

\section{REFERENCES}

Barger, A. J., Cowie, L. L., \& Richards, E. A. 2000, AJ, 119, 2092

Baugh, C. M., Cole, S., Frenk, C. S., \& Lacey, C. G. 1998, ApJ, 498, 504

Bekki, K. \& Shioya, Y. 2000, PASJ, 52, L57

Brinchmann, J. \& Ellis, R. 2000, ApJ, 536, L77

Calzetti, D., Armus, L., Bohlin, R. C., Kinney, A. L., Koornneef, J., \& Storchi-Bergmann, T. 2000, ApJ, 533, 682

Cen, R. 1992, ApJS, 78, 341

Cen, R., Miralda-Escude, J., Ostriker, J. P., \& Rauch, M. 1994, ApJ, 437, L9 
Cen, R. \& Ostriker, J. P. 1992, ApJ, 399, L113

-. 1993, ApJ, 417, 404

-. 1999a, ApJ, 514, 1

—. 1999b, ApJ, 519, L109

—. 2000, ApJ, 538, 83

Cen, R., Ostriker, J. P., Prochaska, J. X., \& Wolfe, A. M. 2003, ApJ, 598, 741

Chapman, S. C., Blain, A. W., Ivison, R. J., \& Smail, I. R. 2003, Nature, 422, 695

Cimatti, A., Daddi, E., Cassata, P., Pignatelli, E., Fasano, G., Vernet, J., Fomalont, E., Kellermann, K., Zamorani, G., Mignoli, M., \& et al. 2003, A\&A, 412, L1

Cohen, J. G. 2002, ApJ, 567, 672

Cole, S., Lacey, C. G., Baugh, C. M., \& Frenk, C. S. 2000, MNRAS, 319, 168

Cole, S., Norberg, P., Baugh, C. M., Frenk, C. S., Bland-Hawthorn, J., Bridges, T., Cannon, R., Colless, M., Collins, C., Couch, W., \& et al. 2001, MNRAS, 326, 255

Connolly, A. J., Szalay, A. S., Dickinson, M. E., SubbaRao, M. U., \& Brunner, R. J. 1997, ApJ, 486, L11

Cowie, L. L., Songaila, A., \& Barger, A. J. 1999, AJ, 118, 603

Daddi, E., Cimatti, A., Renzini, A., Vernet, J., Conselice, C., Pozzetti, L., Mignoli, M., Tozzi, P., Broadhurst, T., de Serego Alighieri, S., \& et al. 2004, ApJ, 600, L127

Daddi, E., Röottgering, H. J. A., Labbe, I., Rudnick, G., Franx, M., Moorwood, A. F. M., Rix, H. W., van der Werf, P. P., \& van Dokkum, P. G. 2003, ApJ, 588, 50

Dickinson, M., Papovich, C., Ferguson, H., \& Budavári, T. 2003a, ApJ, 587, 25

Dickinson, M., Stern, D., Giavalisco, M., Ferguson, H. C., Tsvetanov, Z., Chornock, R., Cristiani, S., Dawson, S., Dey, A., Filippenko, A. V., Moustakas, L. A., Nonino, M., Papovich, C., Ravindranath, S., Riess, A., Rosati, P., Spinrad, H., Vanzella, E., \& et al. 2003b, ApJ, 600, L99

Elston, R., Rieke, G. H., \& Rieke, M. 1988, ApJ, 331, L77 
Ferguson, P. M. H. C., Dickinson, E. D., Giavalisco, M., Steidel, C. C., \& Fruchter, A. 1996, MNRAS, 283, 1388

Fontana, A., Donnarumma, I., Vanzella, E., Giallongo, E., Menci, N., Nonino, M., Saracco, P., Cristiani, S., D’Odorico, S., Poli, F., \& et al. 2003, ApJ, 594, L9

Franx, M., Labbe, I., Rudnick, G., van Dokkum, P. G., Daddi, E., Förster, S., Natascha, M., Moorwood, A., Rix, H.-W., Röttgering, H., van de Wel, A., \& et al. 2003, ApJ, 587, L79

Gardner, J., Katz, N., Hernquist, L., \& Weinberg, D. H. 2003, ApJ, 587, 1

Giavalisco, M., Dickinson, M., Ferguson, H. C., Ravindranath, S., Kretchmer, C., Moustakas, L. A., Madau, P., Fall, M., Gardner, J. P., Livio, M., \& et al. 2004, ApJ, 600, L103

Glazebrook, K., Abraham, R., McCarthy, P., Savaglio, S., Chen, H.-W., Crampton, D., Murowinski, R., Jorgensen, I., Roth, K., Hook, I., Marzke, R., \& Carlberg, R. 2004, submitted (astro-ph/0401037)

Granato, G. L., Lacey, C. G., Silva, L., Bressan, A., Baugh, C. M., Cole, S., \& Frenk, C. S. 2000, ApJ, 542, 710

Hernquist, L. 1993, ApJ, 404, 717

Hernquist, L. \& Springel, V. 2003, MNRAS, 341, 1253

Hu, E. M., McMahon, R. G., \& Cowie, L. L. 1999, ApJ, 522, L9

Hu, E. M. \& Ridgway, S. E. 1994, AJ, 107, 1303

Hughes, D. H., Serjeant, S., Dunlop, J., Rowan-Robinson, M., Blain, A., Mann, R. G., Ivison, R., Peacock, J., Efstathiou, A., Gear, W., Oliver, S., Lawrence, A., Longair, M., Goldschmidt, P., \& Jenness, T. 1998, Nature, 394, 241

Iwata, I., Ohta, K., Tamura, N., Ando, M., Wada, S., Watanabe, C., Akiyama, M., \& Aoki, K. 2003, PASJ, 55, 415

Kauffmann, G., Colberg, J. M., Diaferio, A., \& White, S. D. M. 1999, MNRAS, 303, 188

Kennicutt, R. C. J., Armus, L., Bendo, G., Calzetti, D., Dale, D. A., Draine, B. T., Gordon, C. W. E. K. D., Grauer, A. D., Helou, G., Hollenbach, D. J., Jarrett, T. H., Kewley, L. J., Leitherer, C., Li, A., Malhotra, S., Regan, M. W., Rieke, G. H., Rieke, M. J., Roussel, H., Smith, J.-D. T., Thornley, M. D., \& Walter, F. 2003, PASP, 115, 928 
Kodaira, K., Taniguchi, Y., Kashikawa, N., Kaifu, N., Ando, H., Karoji, H., Ajiki, M., Akiyama, M., Aoki, K., Doi, M., \& et al. 2003, PASJ, 55, L17

Lanzetta, K. M., Yahata, N., Pascarelle, S., Chen, H.-W., \& Fernández-Soto, A. 2002, ApJ, 570,492

Lilly, S. J., Fèvre, O. L., Hammer, F., \& Crampton, D. 1996, ApJ, 460, L1

Madau, P. 1997, in Star Formation Near and Far, ed. S. S. Holt \& G. L. Mundy, Vol. 393 (New York: AIP), 481

Madau, P., Pozzetti, L., \& Dickinson, M. 1998, ApJ, 498, 106

McCarthy, P. J., Persson, S. E., \& West, S. C. 1992, ApJ, 386, 52

Menci, N., Cavaliere, A., Fontana, A., Giallongo, E., \& Poli, F. 2002, ApJ, 575, 18

Menci, N., Cavaliere, A., Fontana, A., Giallongo, E., Poli, F., \& Vittorini, V. 2003, ApJ, in press (astro-ph/03011496)

Meurer, G. R., Heckman, T. M., \& Calzetti, D. 1999, ApJ, 521, 64

Nagamine, K. 2002, ApJ, 564, 73

Nagamine, K., Fukugita, M., Cen, R., \& Ostriker, J. P. 2001a, ApJ, 558, 497

-. 2001b, MNRAS, 327, L10

Nagamine, K., Springel, V., \& Hernquist, L. 2004a, MNRAS, 348, 421

—. 2004b, MNRAS, 348, 435

Nagamine, K., Springel, V., Hernquist, L., \& Machacek, M. 2004, MNRAS, 350, 385

Norman, C., Ptak, A., Hornschemeier, A., Hasinger, G., Bergeron, J., Comastri, A., Giacconi, R., Gilli, R., Glazebrook, K., Heckman, T., Kewley, L., Ranalli, P., Rosati, P., Szokoly, G., Tozzi, P., Wang, J., Zheng, W., \& A. 2004, ApJ, in press (astro$\mathrm{ph} / 0402140)$

Ouchi, M., Shimasaku, K., Furusawa, H., Miyazaki, M., Doi, M., Hamabe, M., Hayashino, T., Kimura, M., Kodaira, K., Komiyama, Y., \& et al. 2003a, ApJ, 582, 60

—. 2003b, ApJ, submitted (astro-ph/0309655)

Pascarelle, S. M., Lanzetta, K. M., \& Fernandez-Soto, A. 1998, ApJ, 508, L1 
Poli, F., Giallongo, E., Fontana, A., Menci, N., Zamorani, G., Nonino, M., Saracco, P., Vanzella, E., Donnarumma, I., Salimbeni, S., \& et al. 2003, ApJ, 593, L1

Rhoads, J. E. \& Malhotra, S. 2001, ApJ, 563, L5

Rudnick, G., Rix, H.-W., Franx, M., Labbe, I., Blanton, M., Daddi, E., Förster, S., Natascha, M., Moorwood, A., Röttgering, H., Trujillo, I., \& et al. 2003, ApJ, 599, 847

Ryu, D., Ostriker, J. P., Kang, H., \& Cen, R. 1993, ApJ, 414, 1

Sawicki, M. J., Lin, H., \& Yee, H. K. C. 1997, AJ, 113, 1

Smail, I., Ivision, R. J., \& Blain, A. W. 1997, ApJ, 490, L5

Smail, I., Owen, F. N., Morrison, G. E., Keel, W. C., Ivison, R. J., \& Ledlow, M. J. 2002, ApJ, 581, 844

Somerville, R. S., Moustakas, L. A., Mobasher, B., Gardner, J. P., Cimatti, A., Conselice, C., Daddi, E., Dahlen, T., Dickinson, M., Eisenhardt, P., Lotz, J., Papovich, C., Renzini, A., \& Stern, D. 2004, ApJ, 600, L135

Somerville, R. S., Primack, J. R., \& Faber, S. M. 2001, MNRAS, 320, 504

Spergel, D., Verde, L., Peiris, H. V., Komatsu, E., Nolta, M. R., Bennett, C. L., Halpern, M., Hinshaw, G., Jarosik, N., Kogut, A., \& et al. 2003, ApJS, 148, 175

Springel, V. \& Hernquist, L. 2002, MNRAS, 333, 649

-. 2003a, MNRAS, 339, 289

-. 2003b, MNRAS, 339, 312

Springel, V., Yoshida, N., \& White, S. D. M. 2001, New Astronomy, 6, 79

Steidel, C. C., Adelberger, K. L., Giavalisco, M., Dickinson, M., \& Pettini, M. 1999, ApJ, 519,1

Takagi, T., Vansevicius, V., \& Arimoto, N. 2003, PASJ, 55, 385

Taniguchi, Y., Ajiki, M., Murayama, T., Nagao, T., Veilleux, S., Sanders, D. B., Komiyama, Y., Shioya, Y., Fujita, S., Kakazu, Y., \& et al. 2003, ApJ, 585, L97

Tecza, M., Baker, A., Davies, R., Genzel, R., Lehnert, M. D., Eisenhauer, F., Lutz, D., Nesvadba, N., Seitz, S., Tacconi, L. J., Thatte, N. A., Abuter, R., \& Bender, R. 2004, ApJ, in press (astro-ph/0403264) 
Treyer, M. A., Ellis, R. S., Millard, B., Donas, J., \& Bridges, T. J. 1998, MNRAS, 300, 303

Yoshida, N., Bromm, V., \& Hernquist, L. 2003, ApJ, submitted (astro-ph/0310443) 
Table 1. Simulations

\begin{tabular}{cccccc}
\hline \hline Run & $L_{\text {box }}\left[h^{-1} \mathrm{Mpc}\right]$ & $N_{\text {mesh/ptcl }}$ & $m_{\text {DM }}\left[h^{-1} M_{\odot}\right]$ & $m_{\text {gas }}\left[h^{-1} M_{\odot}\right]$ & $\Delta \ell\left[h^{-1} \mathrm{kpc}\right]$ \\
\hline TVD: N864L11 $^{a}$ & 11.0 & $864^{3}$ & $1.1 \times 10^{6}$ & $2.7 \times 10^{4}$ & 12.7 \\
TVD: N864L22 $^{a}$ & 22.0 & $864^{3}$ & $8.9 \times 10^{6}$ & $2.2 \times 10^{5}$ & 25.5 \\
TVD: N768L25 $^{b}$ & 25.0 & $768^{3}$ & $2.0 \times 10^{7}$ & $3.4 \times 10^{5}$ & 32.6 \\
SPH: Q5 $^{c}$ & 10.0 & $324^{3}$ & $2.1 \times 10^{6}$ & $3.3 \times 10^{5}$ & 1.2 \\
SPH: D5 $^{c}$ & 33.75 & $324^{3}$ & $8.2 \times 10^{7}$ & $1.3 \times 10^{7}$ & 4.2 \\
SPH: G6 $^{c}$ & 100.0 & $486^{3}$ & $6.3 \times 10^{8}$ & $9.7 \times 10^{7}$ & 5.3 \\
\hline
\end{tabular}

Note. - Parameters of some of the simulations on which this study is based. The quantities listed are as follows: $L_{\text {box }}$ is the simulation box-size, $N_{\text {mesh/ptcl }}$ is the number of the hydrodynamic mesh points for TVD or the number of gas particles for SPH, $m_{\mathrm{DM}}$ is the dark matter particle mass, $m_{\text {gas }}$ is the mass of the baryonic fluid elements in a grid cell for TVD or the masses of the gas particles in the SPH simulations. Note that the TVD uses $432^{3}\left(384^{3}\right)$ dark matter particles for N864 (N768) runs. $\Delta \ell$ is the size of the resolution element (cell size in TVD and gravitational softening length in SPH in comoving coordinates; for proper distances, divide by $1+z)$. The upper indices on the run names correspond to the following sets of cosmological parameters: $\left(\Omega_{\mathrm{M}}, \Omega_{\Lambda}, \Omega_{b}, h, n, \sigma_{8}\right)=(0.29,0.71,0.047,0.7,1.0,0.85)$ for (a), $(0.3,0.7,0.035,0.67,1.0,0.9)$ for $(\mathrm{b})$, and $(0.3,0.7,0.04,0.7,1.0,0.9)$ for $(\mathrm{c})$. 

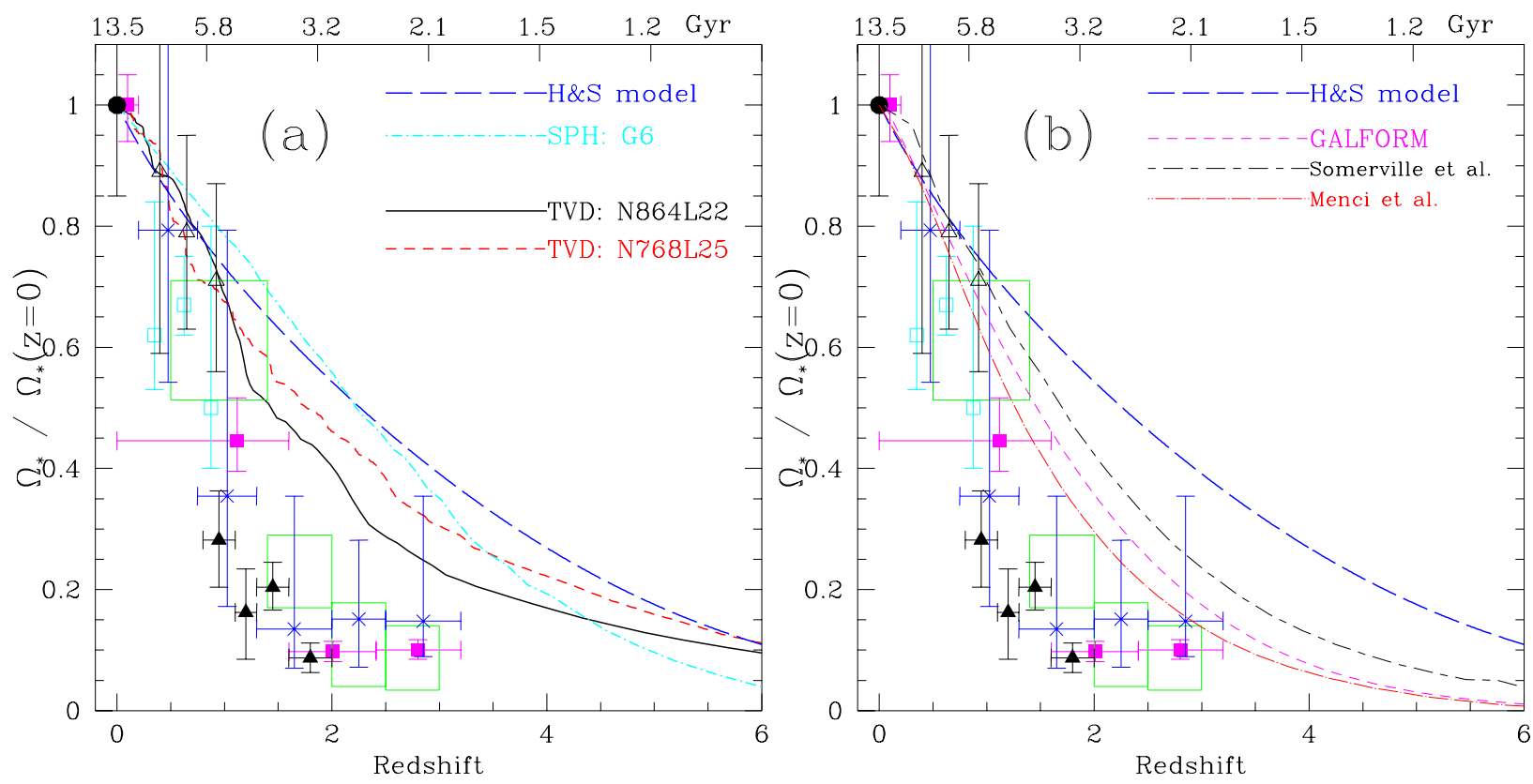

Fig. 1.- Development of stellar mass density as a function of redshift. Panel (a): The black solid line is from the most recent TVD N864L22 run (consistent with the not shown N864L11 run), and the red short-dashed line is from the TVD N768L25 run which was used in Nagamine et al. (2001a). The blue long-dashed line is the analytical model of Hernquist \& Springel (2003), and the cyan dot-long-dashed line is the result from the SPH 'G6'-run. The observational data points are, from low to high redshift, Cole et al. (2001, black filled circle at $z=0)$, Brinchmann \& Ellis (2000, cyan open squares at $z=0.5-1)$, Cohen (2002, black open triangles at $z=0.5-1$ ), Glazebrook et al. (2004, black filled triangles at $z=1-2$ ), Rudnick et al. (2003, magenta filled squares at $z=0-3$ ), and Fontana et al. (2003, blue open crosses at $z=0.5-3)$. The four green boxes are from Dickinson et al. (2003a) which show the range of systematic uncertainty introduced by varying the metallicity and star formation histories of the mass-fitting model they used to derive the stellar mass density. All the data points are normalized to the local estimate by Cole et al. (2001) following Rudnick et al. (2003). Panel (b): Observational data points and the H\&S model are the same as panel (a). The results of two semi-analytic models by Somerville et al. (2001, black long-short-dashed line), Granato et al. (2000, magenta short-dashed line; GALFORM), and Menci et al. (2002, red dot-long-dashed line) are shown. The model of Somerville et al. (2001) is the 'accelerated quiescent' model which was used in Somerville et al. (2004), and the model of Menci et al. (2002) is the 'no-burst' model that was used in Poli et al. (2003) and Fontana et al. (2003) for comparison with their data. 

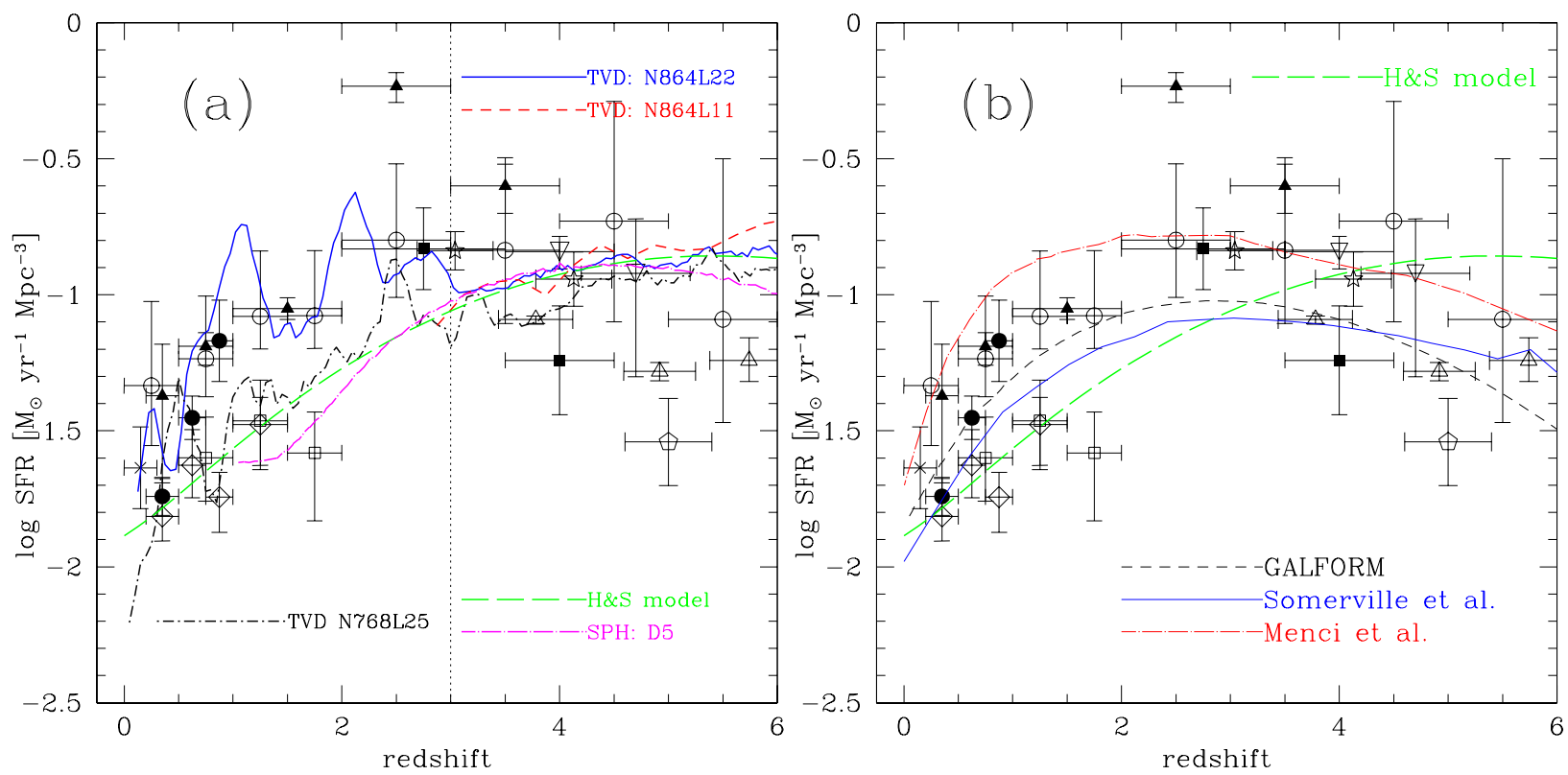

Fig. 2.- Star formation rate density as a function of redshift. Panel (a): The lines correspond to TVD N864L22 (solid blue line), TVD N864L11 (red short-dashed line; stopped at $z=3$ owing to its small box-size), TVD N768L25 (black dot long-dashed line), SPH 'D5'run (magenta dot long-dashed line), SPH 'G6'-run (cyan short-dash long-dash line), and the model of Hernquist \& Springel (2003, green long-dashed line) as given by equation (1). The simulation results shown in this figure are extracted directly from the runs, without any further adjustments. The result of the SPH Q5-run is not shown here because it follows the Hernquist \& Springel (2003) model line very closely at $z \geq 3$. The sources of the (dust corrected) observational data points are: Lilly et al. (1996, filled circles), Ferguson et al. (1996); Madau (1997, filled squares), Connolly et al. (1997, open squares), Sawicki et al. (1997, filled triangles), Treyer et al. (1998, open cross), Pascarelle et al. (1998, open circles), Cowie et al. (1999, open diamond), Steidel et al. (1999, open stars at $z=3,4)$, Iwata et al. (2003, open pentagon at $z=5$ ), Ouchi et al. (2003b, open inverted triangle at $z=4,5$ ), and Giavalisco et al. (2004, open triangles at $z=3-6$ ). Panel (b): H\&S model (green long-dashed line), semi-analytic models of Somerville et al. (2001, blue solid line), Granato et al. (2000, black short-dashed line), and Menci et al. (2002) are compared with observations. The model of Somerville et al. (2001) is the 'accelerated quiescent' model which was used in Somerville et al. (2004), and the model of Menci et al. (2002) is the 'no-burst' model that was used in Poli et al. (2003) and Fontana et al. (2003) for comparison with their data. Both simulations and H\&S model has a peak of SFR at $z=5-7$, whereas the semi-analytic models have a peak at $z=2-4$. 